\title{
The mTORC2/Akt/NFkB Pathway-Mediated Activation of TRPC6 Participates in Adriamycin-Induced Podocyte Apoptosis
}

\author{
Hai-Tao Zhang ${ }^{\mathrm{a}}$ Wei-Wei Wang ${ }^{\mathrm{b}} \quad$ Li-Hong Ren $^{\mathrm{a}} \quad$ Xia-Xia Zhao ${ }^{\mathrm{a}} \quad$ Zhi-Hui Wang $^{\mathrm{a}}$ \\ De-Li Zhuang ${ }^{\text {a }}$ Yun-Nuo Baia \\ aDepartment of Pediatrics, 'bepartment of anesthesiology, Harbin Medical University Second Hospital, \\ Harbin, Heilongjiang, PR China
}

\section{Key Words \\ Podocyte apoptosis $•$ Adriamycin (ADR) $・$ TRPC6 $・$ mTOR $・$ Akt $・ N F k B$}

\begin{abstract}
Background/Aims: Although increased expression and gain function of transient receptor potential cation channel 6 (TRPC6) has been associated with the pathogenesis of some proteinuric glomerular diseases, it remains elusive how TRPC6 participates in the process of podocyte damage. Methods: The potential signaling responsible for TRPC6 activation was investigated using immunoblot assays in an in vitro podocyte injury model induced by Adriamycin (ADR). Podocyte apoptosis was measured using FITC-conjugated Annexin V and Propidium Iodide staining. The channel activity of TRPC 6 was assessed using the $\mathrm{Ca}^{2+}$ influx assay. Results: Increase of TRPC6 expression was detected in ADR-treated podocytes, and TRPC6 knockdown significantly decreased ADR-induced podocytes apoptosis. Following ADR treatment, phospho-mTOR ${ }^{\text {Ser2481 }}$ and phospho-Akt ${ }^{\text {Ser473 }}$ was significantly increased in a time-dependent manner, whereas phospho-mTOR ${ }^{\mathrm{Ser} 2448}$ and phospho-p70S6K ${ }^{\mathrm{Th} 3389}$ showed no change. ADR-induced apoptosis was prevented by ku0063794 (a dual mTOR complexes inhibitor), not by rapamycin (a specific mTORC1 inhibitor). Furthermore, nuclear translocation of NFKB/p65 was detected in ADR-treated podocytes, which was prevented by an Akt inhibitor triciribine. Of note, NFKB inhibitor PDTC prevented ADR-induced increase of TRPC6, and decreased ADR-induced apoptosis. We found that Akt activation and NFKB nuclear translocation was significantly inhibited by knockdown of mTORC2 protein Rictor, not by mTORC1 protein Raptor. In comparison with control, the $\mathrm{Ca}^{2+}$ influx was significantly increased in ADR-treated podocytes, which was remarkably prevented by TRPC 6 knockdown. ADR-induced increase of TRPC6 channel activity was dramatically prevented by ku0063794, but not by rapamycin. Additionally, knockdown of Rictor, not Raptor, prevented ADR-induced increase of the $\mathrm{Ca}^{2+}$ influx. Moreover, the application of NFKB inhibitor PDTC also prevented the $\mathrm{Ca}^{2+}$ influx in ADRtreated podocytes. Conclusions: Our findings revealed that the mTORC2/Akt/NFkB pathwaymediated activation of TRPC6 participates in ADR-induced podocyte apoptosis.
\end{abstract}




\section{Introduction}

Proteinuria is one of the most important clinical manifestations in children nephrotic syndrome. Glomerular visceral epithelial cells, also termed as podocytes, are the terminally differentiated and highly specialized cells with a large cell body extending primary and secondary foot processes (FPs) that are linked by the slit diaphragm (SD) necessary for maintaining normal glomerular filtration function [1]. The abnormalities of podocytes, particularly SD, play a key role in the development of proteinuria. Increasing evidences demonstrated that podocyte loss due to apoptosis/death or detachment from glomerular basement membrane is involved in progressive glomerulosclerosis [2]. Additionally, damage to the glomerular filter, in particular to podocytes and SD, is of crucial importance in the pathogenesis of proteinuric diseases. Transient receptor potential cation channel 6 (TRPC6), a non-selective cation channel, has been identified as a novel podocyte SD protein $[3,4]$. Mutations of TRPC6 cause increase of its channel activity and podocyte damage, leading to autosomal dominant focal segmental glomerulosclerosis (FSGS) [3]. TRPC6, ubiquitously expressed in many types of cells, can affect intracellular $\mathrm{Ca}^{2+}$ level through regulating influx of $\mathrm{Ca}^{2+}[4,5]$, while excessive intracellular $\mathrm{Ca}^{2+}$ could lead to cells apoptosis $[4,6,7]$. Interestingly, increased expression of TRPC6 was found in many acquired human proteinuric glomerular diseases, including non-familial FSGS, minimal change disease and membranous nephropathy $[8,9]$. Notably, the expression and channel function of TRPC6 is increased in some in vitro podocyte damaged model. Angiotensin II (AngII) results in podocyte apoptosis by increasing TRPC6 expression and intracellular $\mathrm{Ca}^{2+}$ level, which is mediated by ERK activation-induced NFKB nuclear translocation $[10,11]$. Abundance of TRPC6 is also increased through NADPH oxidase-mediated ROS signaling pathway in response to puromycin aminonucleosideinduced podocyte injury $[11,12]$. In high glucose-induced podocyte damage, activation of TRPC6 is mediated by the Wnt/ $\beta$-catenin signaling pathway [13]. Nevertheless, it remains elusive how TRPC6 participates in the pathogenesis of glomerular podocyte injury.

Recently, it was reported that the inhibition of mammalian target of rapamycin (mTOR), can regulate TRPC6 expression in cultured normal podocytes $[14,15]$. The serine/threonine kinase mTOR forms two distinct functional complexes, mTORC1 and mTORC2 [16]. The mTORC1 complex including at least three additional components, Raptor, mLST8, and FKBP12, is rapamycin-sensitive since the interaction of the rapamycin and FKBP12 interferes with the binding of Raptor to mTOR [17]. Nevertheless, the mTORC2 consists of mLST8, Sin1, and Rictor, and is rapamycin-insensitive since the Rictor-mTOR complex is not able to bind the FKBP12-rapamycin conjugate [17]. Recent studies revealed that physiologic levels of mTORC1 and mTORC2 activity play key roles in maintaining podocyte and glomerular functions. Aberrant activation of mTORC1 or loss of mTORC2 activity in podocytes may be involved in the pathogenesis of glomerular diseases [18]. In this study, we focused on the distinct role of mTOR signaling, and investigated how TRPC6 contributes to Adriamycin (ADR)-induced podocyte damage. We provide evidence that the functional activation of TRPC6 is mediated by the mTORC2/Akt/NFKB pathway, which may participate in ADRinduced podocyte apoptosis.

\section{Materials and Methods}

\section{Antibodies}

The following antibodies were used: rabbit anti-TRPC6 (ab12249), mouse anti-beta actin (ab6276), mouse anti-mTOR (ab87540), and rabbit anti-phospho-mTOR ${ }^{\text {Ser2448 }}$ (ab109268) or phospho-mTOR ${ }^{\text {Ser2481 }}$ (ab137133, Abcam), mouse anti-phospho-p70S6K ${ }^{\text {Thr389 }}$ (\#9206), rabbit anti-p70S6K (\#9202), rabbit antiphospho-Akt $^{\text {Ser473 }}$ (\#4060), and mouse anti-Akt antibody (\#2920, Cell Signaling), mouse anti-NFkB/p65 (sc-8008), rabbit anti-Histone H3 (sc-10809), rabbit anti-caspase3 antibody (sc-7148), rabbit anti-cleaved caspase 3 antibody (sc-22171), and rabbit anti-IкB $\alpha$ (sc-371, Santa Cruz), HRP-conjugated goat anti-rabbit or mouse IgG (32230, 32260; Thermo Scientific). 


\section{Cellular Physiology Cell Physiol Biochem 2016;40:1079-1093 \begin{tabular}{l|l|l} 
and Biochemistry & $\begin{array}{l}\text { DOI: 10.1159/000453163 } \\
\text { Published online: December 14, } 2016\end{array}$ & $\begin{array}{l}\text { (c) } 2016 \text { The Author(s). Published by S. Karger AG, Basel } \\
\text { www.karger.com/cpb }\end{array}$
\end{tabular} Zhang et al.: TRPC6 Signaling in ADR-Induced Podocyte Apoptosis}

Cell culture and treatment

Mouse podocyte clone was a kind gift from

Prof. Peter Mundel. Podocyte was cultured at $33^{\circ} \mathrm{C}$ in RPMI 1640 media (Catalog number: 61870-036; Invitrogen) supplemented with 10\% fetal calf serum (Catalog number: 10438-026; Gibco), $100 \mathrm{U} / \mathrm{ml}$ of Penicillin/Streptomycin (Catalog number: 15140-122; Gibco), and $10 \mathrm{U} /$ $\mathrm{ml}$ of recombinant mouse $\gamma$-interferon (Catalog number: 11276905001; Sigma Aldrich). To induce apoptosis, podocyte was treated with $0.5 \mu \mathrm{g} / \mathrm{ml}$ of Adriamycin (ADR; D1515, Sigma Aldrich) for the indicated time periods. In the experiments using the inhibitor rapamycin (10 nM; Catalog number: 553210;

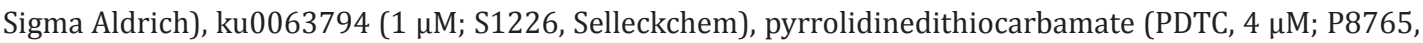
Sigma Aldrich), or triciribine (20 $\mu$ M; Catalog number: 124038; Sigma Aldrich), podocyte was pre-treated with the above inhibitor for $30 \mathrm{~min}$. ADR was then added in the presence of inhibitors (Table 1).

\section{Knockdown assay}

Specific validated siRNA targeting mouse TRPC6 (sc-42673), Raptor (sc-108002), or Rictor (sc61479) and non-targeted control siRNA (sc-37007) was used for knockdown assay (Santa Cruz). To increase transfection efficiency, proliferative podocytes were used in this study. Podocyte was cultured at $33^{\circ} \mathrm{C}$ in 6-well plate, and each well 100 pmol of siRNA duplexes was introduced using $10 \mu \mathrm{l}$ of RNAiMAX Reagent according to manufacturer's instruction (Catalog number: 13778-075; Invitrogen). After 48 hours of transfection, cells were collected for Immunoblot assay and Calcium Flux studies.

\section{Apoptosis detection assay}

Apoptotic cells were labeled by using FITC-conjugated Annexin V and Propidium Iodide (Catalog number: 556547; BD Biosciences) in live podocytes. Briefly, 1 x $10^{5}$ cells were washed twice with cold phosphate-buffered saline by centrifugation at 1,000 rpm for 5 mins. Cell pellets were re-suspended in $1 \mu \mathrm{g} / \mathrm{ml}$ of FITC-Annexin V for $30 \mathrm{~min}$ on ice, and then $5 \mu \mathrm{l}$ of Propidium Iodide $(50 \mu \mathrm{g} / \mathrm{ml}$ ) was added immediately prior to detection with flow cytometry (FACScan).

\section{Real time PCR}

Total RNA was extracted with Trizol (Catalog number: 15596-018; Invitrogen), and $1.5 \mu \mathrm{g}$ of RNA was used for reverse transcription to make complementary DNA (cDNA) by using the First-strand cDNA synthesis kit (Catalog number: 12328-032; Invitrogen). Real time PCR for TRPC6 expression was performed in $25 \mu \mathrm{l}$ reaction volume containing $2 \mu \mathrm{l}$ of cDNA, $1 \mu \mathrm{l}$ of $5 \mu \mathrm{M}$ specific PCR primers (forward: 5'-tggtgcggaagatgctagaa-3'; reverse: 5'-aaagcatcccaactcgaga-3'), $12.5 \mu \mathrm{l}$ of 2x SYBR Green Master Mix (Catalog number: 4309155; Applied Biosystems). The level of TRPC6 was normalized to the housekeeping gene $\beta$-actin (forward: 5'-agccatgtacgtagccatcc-3'; reverse: 5' - gctgtggtggtgaagctgta-3'), and the fold change was compared.

\section{Immunoblot assay}

The RIPA buffer [25 mM Tris- $\mathrm{HCl} \mathrm{pH}$ 7.6, $150 \mathrm{mM} \mathrm{NaCl}$ 1\% NP-40, 1\% sodium deoxycholate (DOC), $0.1 \%$ sodium dodecyl sulfate (SDS), and $10 \%$ glycerol] was used to extract total cellular protein. The protease and phosphatase cocktail inhibitors (Catalog number: 05892970001, 4906845001; Roche) were added immediately prior to lysing cells. Nuclear Protein Extraction Kit (ab113474, Abcam) was used for evaluation of NFKB translocation. The $100 \mu \mathrm{g}$ of protein was electrophoresed on 7.5 or $12.5 \%$ SDS-PAGE. Protein was then semi-transferred to nitrocellulose membranes (GE Healthcare Bioscience). The membranes were blocked for 1 hour in $0.05 \%$ Tween-20 Tris-buffered saline solution (TTBS) containing 2\% BSA/3\% non-fat milk. Membranes were incubated overnight at $4{ }^{\circ} \mathrm{C}$ with the indicated primary antibodies. After 5 washes with TTBS, HRP conjugated secondary antibody was applied for 1 hour. After washes with TTBS, blots were developed with an ECL chemiluminescence detection kit (Catalog number: 32109; Pierce). The specific band was scanned and quantified with Image J. 


\section{Cellular Physiology Cell Physiol Biochem 2016;40:1079-1093 \\ and Biochemistry Published online: December 14, $2016 \quad \begin{aligned} & \text { DOI: 10.1159/000453163 } 2016 \text { The Author(s). Published by S. Karger AG, Basel } \\ & \text { www.karger.com/cpb }\end{aligned}$ \\ Zhang et al.: TRPC6 Signaling in ADR-Induced Podocyte Apoptosis}

\section{Calcium Flux studies}

The fluorescence intensity of free $\mathrm{Ca}^{2+}$ indicator Fluo-3AM (F1241, Invitrogen) was used to measure the level of intracellular free $\mathrm{Ca}^{2+}$. Podocytes were seeded in 96-well plate, and incubated with $5 \mu \mathrm{M}$ of Fluo-3AM for 30 mins at $37^{\circ} \mathrm{C}$. After 3 times washes with PBS, the baseline of intracellular free $\mathrm{Ca}^{2+}$ level was measured using FLX800 spectrophotofluorometer (BioTek) equipped with a filter of $480 \mathrm{~nm}$ excitation and $510 \mathrm{~nm}$ emission. Of note, two wells with PBS alone were used as blank control. Cells were then stimulated by addition of $10 \mu \mathrm{M}$ of hyperforin (H1792, Sigma Aldrich) to initiate activation of TRPC6, and the intracellular free $\mathrm{Ca}^{2+}$ level was recorded for 2 minutes at a 15-second interval. The intracellular free $\mathrm{Ca}^{2+}$ level from three independent experiments was averaged, and the relative fluorescence intensity to the baseline was compared.

\section{Statistical analysis}

Data are present as mean \pm SD. Statistical analysis was performed with Prism 6 (GraphPad Software, Inc.) by using One-Way ANOVA for comparing multiple time-points or Two-Way ANOVA for comparing multiple groups. A p value equal to or less than 0.05 was considered as significant difference.

\section{Results}

Induction of TRPC6 is involved in ADR-mediated podocyte apoptosis

As reported previously [19], we applied $0.5 \mu \mathrm{g} / \mathrm{ml}$ of ADR to cultured mouse podocyte cell line. Apoptotic cells were labeled with FITC-Annexin V and propidium iodide, and the percentage of apoptotic cells was then assessed using flow cytometry. As compared with control, podocyte apoptosis was significantly induced at 24 (14.58 \pm 2.86 vs $2.98 \pm 1.02$; p $<0.001)$ and $48(23.03 \pm 2.57$ vs $2.98 \pm 1.02 ; \mathrm{p}<0.001)$ hours following ADR application (Fig. 1A). TRPC6, a receptor-activated non-selective calcium permeant cation channel, plays an important role in the process of podocyte injury through increasing the entry of $\mathrm{Ca}^{2+}$ to cells [6]. Results from real time RT-PCR show that ADR significantly $(p<0.05)$ increased the mRNA level of TRPC6 at 24 and 48 hours (Fig. 1B). Consistently, induction of TRPC6 at protein level was also detected $(\mathrm{p}<0.01)$ at 24 and 48 hours in ADR-treated podocytes (Fig. 1C). To verify the role of TRPC6 in ADR-treated podocyte, TRPC6 expression was successfully downregulated using the specific TRPC6 siRNA (Fig. 1D,E). The effects of TRPC6 knockdown on cellular apoptosis were then assessed in both wild type and ADR-treated podocytes. TRPC6 knockdown showed no significant effect on apoptosis in wild type podocytes (Fig. $1 \mathrm{~F})$. Nevertheless, the percentage of apoptotic cells was decreased significantly (8.83 \pm 1.12 vs $12.38 \pm 1.49 ; \mathrm{p}<0.01$ ) by TRPC6 knockdown in ADR-treated podocytes although the apoptosis in ADR-treated podocytes with TRPC6 knockdown was still higher (8.83 \pm 1.12 vs $2.98 \pm 1.02 ; \mathrm{p}<0.01$ ) than that in wild-type cells. Of note, control siRNA showed no effects $(12.55 \pm 1.06$ vs $12.38 \pm 1.49 ; \mathrm{p}>0.05)$ on ADR-induced apoptosis (Fig. $1 \mathrm{~F})$. Caspase-3 is a critical executioner of apoptosis in many types of cells. We assessed the activated caspase 3 level using immunoblot assay by detecting the cleaved caspase3 in ADR-treated podocytes. Our data showed that the cleaved caspase 3 abundance increased significantly $(\mathrm{p}<0.05)$ at 24 hours in ADR-treated podocytes, which was prohibited by TRPC6 knockdown (Fig. 1G).

The mTORC2 activation is related to ADR-induced podocyte apoptosis

Due to distinct components and substrates, mTOR complexes are categorized into two complexes, mTOR complex 1 (mTORC1) and mTOR complex 2 (mTORC2). The mTORC1 activation predominantly at Ser2448 leads to the phosphorylation of its downstream target p70S6K, while the mTORC2 activation predominantly at Ser2481 leads to the phosphorylation of Akt at Ser473 $[14,17]$. In ADR-treated podocytes, the phosphorylation of mTOR at Ser2481 increased significantly in a time-dependent manner, whereas the phosphorylation of mTOR at Ser2448 showed no change (Fig. 2A), indicating that the mTORC2 complex is activated following ADR treatment in cultured podocytes. In consistent, we detected a time-dependent increase of phospho-Akt ${ }^{\text {Ser473}}$, but not phospho-p70S6K ${ }^{\text {Thr389 }}$ in ADR-treated podocytes (Fig. 


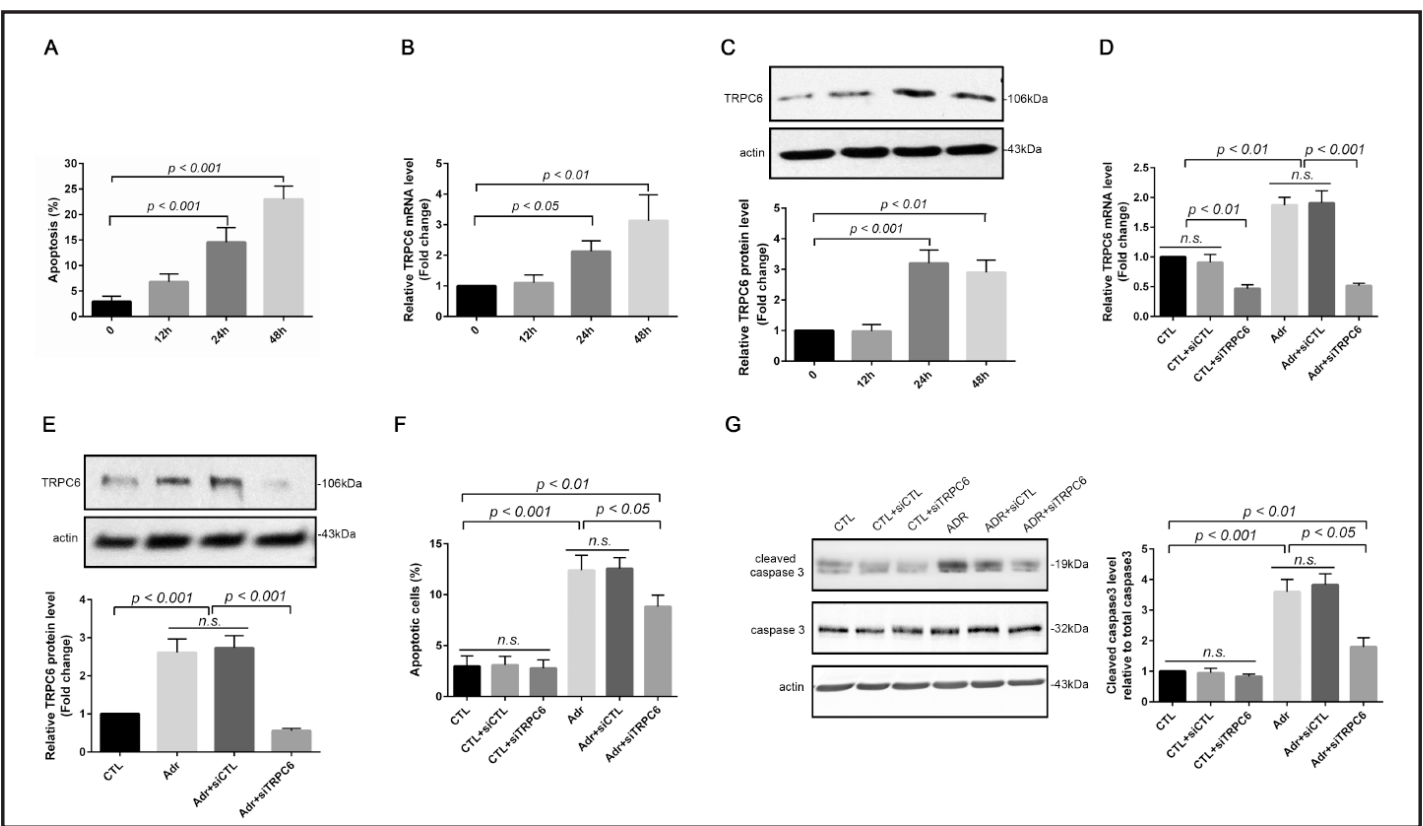

Fig. 1. TRPC6 upregulation is related to ADR-induced podocyte apoptosis. A - C: Podocytes were treated with Adriamycin $(0.5 \mu \mathrm{g} / \mathrm{ml})$ for the indicated time periods. Cellular apoptosis was assessed using FITC-Annexin $\mathrm{V}$ and propidium iodide staining by flow cytometry (A). The abundance of TRPC6 was evaluated using real time RT-PCR (B) and Western blot (C) in ADR-treated podocytes. D - G: The siRNA targeting to mouse TRPC6 (siTRPC6) was introduced using RNAiMAX to knockdown TRPC6 expression. The control siRNA (siCTL) has no any significant sequence similarity to mouse gene sequences. 24 hours after transfection, cells were treated with Adriamycin (ADR, $0.5 \mu \mathrm{g} / \mathrm{ml}$ ) or cultured media alone as control (CTL) for another 24 hours. The level of TRPC6 was assessed using real time RT-PCR (D) and Western blot (E), respectively. Effect of TRPC6 knockdown on cellular apoptosis was examined (F). The cleaved caspase3 level was assessed using immunoblot assay (G). A, F: $\mathrm{n}=4$ independent experiments and triplicates were used for each experiment. B - E and G: triplicates were used, and the representative blots were provided. n.s.: no significant difference.

2B). To assess the distinct role of the two different mTOR complexes in ADR-induced podocyte apoptosis, the cultured podocytes were exposed to rapamycin, an inhibitor of mTORC1, and ku0063794, a dual inhibitor of both mTORC1 and mTORC2. As the data showed, rapamycin application dramatically inhibited the phosphorylation of mTOR at Ser2448 and p70S6K at Thr389, while showed no effects on the phosphorylated mTOR at Ser2481 and Akt at Ser473 (Fig. 2C). The level of phospho-mTOR at Ser2448 and phospho-p70S6K at Thr 389 was remarkably decreased by ku0063794. Of note, ADR-induced upregulation of mTOR at Ser2481 and Akt activation at Ser473 was significantly prevented by ku0063794 (Fig. 2C). We also evaluated the effects of distinct mTOR complexes inhibition on ADR-induced podocyte injury. Firstly, our results showed that there was no significant difference of cellular apoptosis between wild-type podocytes and wild-type podocytes treated with rapamycin or ku0063794 alone (Fig. 2D). Compared to control, ADR significantly induced podocyte apoptosis $(12.7 \pm 1.51$ vs $4.2 \pm 0.71 ; \mathrm{p}<0.001)$ at 24 hours following treatment. In comparison to ADR alone, the administration of ku0063794 significantly decreased ADRinduced podocyte apoptosis ( $7.5 \pm 1.41$ vs $12.7 \pm 1.51$; $\mathrm{p}<0.001$ ), while rapamycin treatment displayed no significant effects (11.4 \pm 1.02 vs $12.7 \pm 1.51)$ (Fig. 2D).

\section{$N F K B$ activation is associated with TRPC6 induction and cellular apoptosis in ADR-treated podocyte}

$\mathrm{NF} \kappa \mathrm{B}$ is a protein complex that controls transcription of DNA, cytokine production and cell survival $[20,21]$. It has been reported that NFKB is involved in up-regulation of TRPC6 in angiotensin II-induced podocyte damage [10]. Non-activated NFKB binds to I $\mathrm{B}$ proteins, 


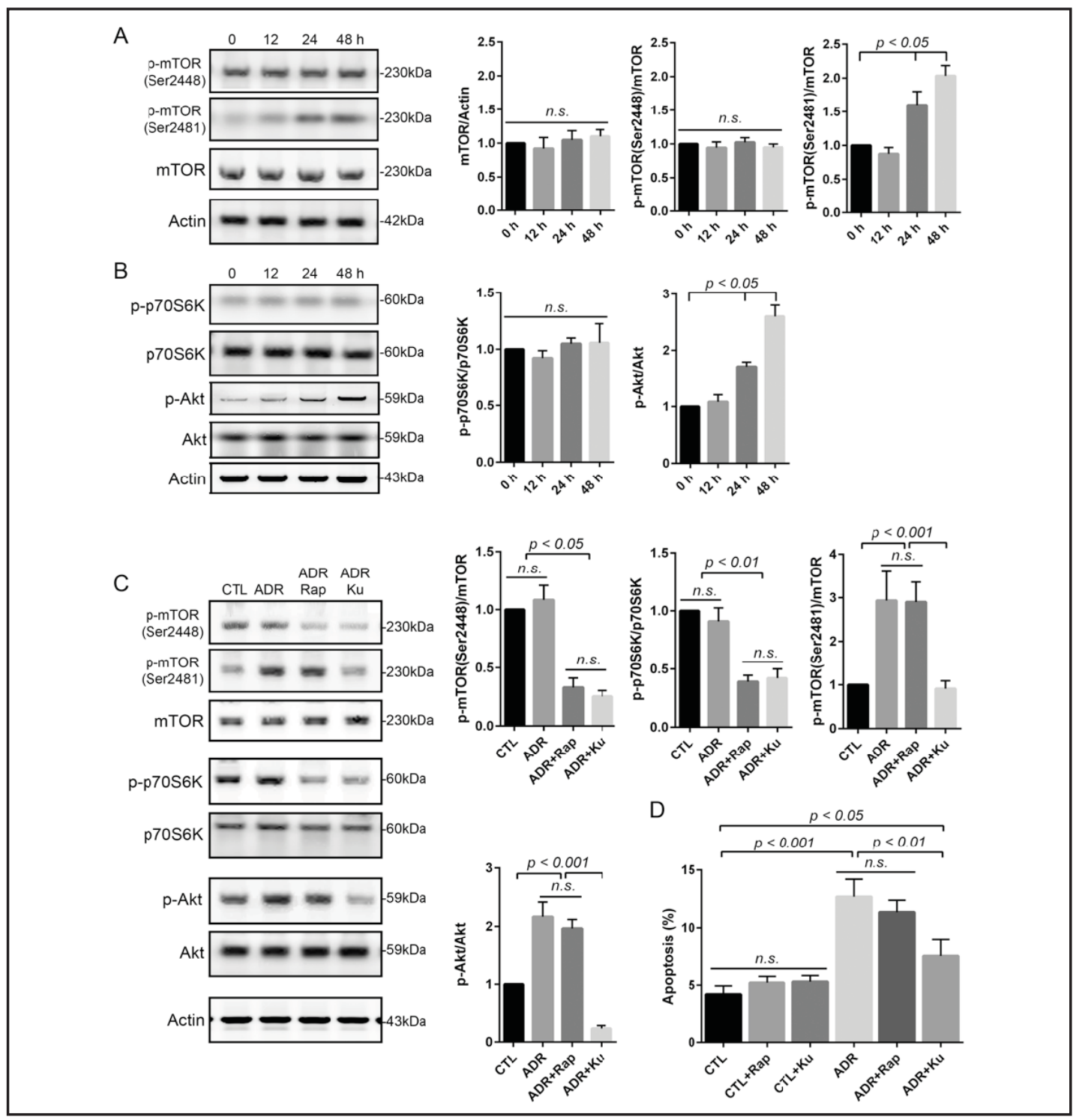

Fig. 2. The mTORC2 activation is involved in Adr-induced podocyte apoptosis. A, B. Podocytes were treated with Adriamycin (ADR, $0.5 \mu \mathrm{g} / \mathrm{ml}$ ) for the indicated time periods. Total cellular protein was extracted and immunoblot assay was performed to evaluate the activation level of mTOR complexes (A) as well as p70S6K and Akt (B), respectively. C, D. Podocytes were pre-treated with rapamycin (Rap; $10 \mathrm{nM}$ ) or ku006394 (Ku; 1 $\mu \mathrm{M}$ ) for 1 hour. ADR was then added (final concentration $0.5 \mu \mathrm{g} / \mathrm{ml}$ ) and incubated for 24 hours in the presence of the indicated inhibitors. Immunoblot assay was performed to examine the level of phospho-mTORSer2448, phospho-mTOR ${ }^{\text {Ser2481, }}$, phospho-p70S6K, and phospho-Akt (C). Effect of rapamycin and ku0063794 on podocyte apoptosis was assessed using FITC-Annexin V and propidium iodide staining by flow cytometry (D). A - C: three individual experiments were performed, and the representative blots were provided. D: $\mathrm{n}=$ 4 independent experiments, and triplicates were used for each group. n.s.: no significant difference.

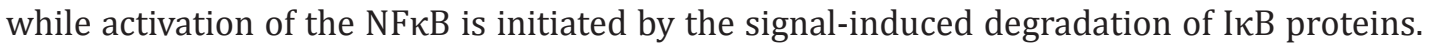
With the degradation of $I \kappa \mathrm{B}$, the NFKB complex is then released and thus enters the nucleus where it can turn on the expression of specific genes that have DNA-binding sites for NFkB [20]. In this study, nuclear fraction was obtained from ADR-treated podocytes, evidenced by the absence of GAPDH using immunoblot assay (Fig. 3A). The abundance of NFkB/p65 in the nuclear fractions was increased significantly in a time-dependent manner (Fig. 3A), whereas the cytosolic IкB- $\alpha$ was decreased remarkably (Fig. 3B). To evaluate the role of nuclear 


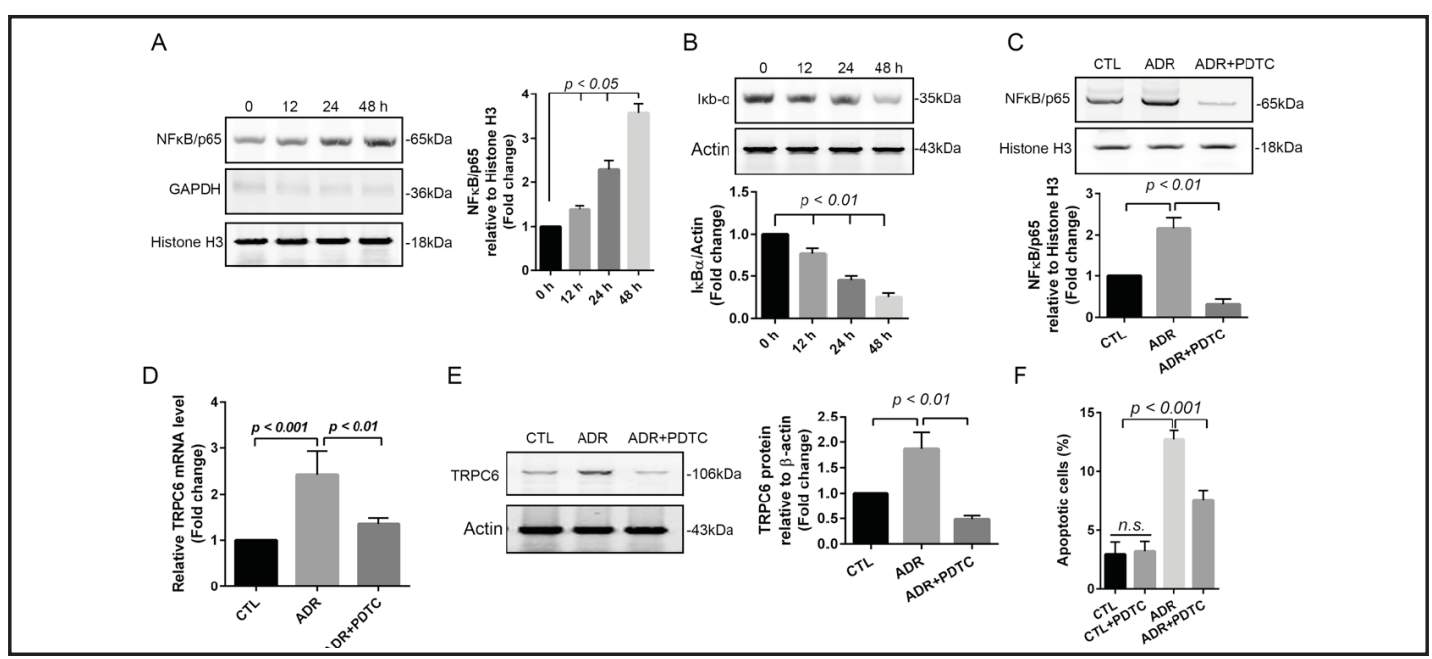

Fig. 3. NFKB activation is responsible for TRPC6 induction and cellular apoptosis in Adr treated podocytes. A, B. Podocytes were treated with Adriamycin (ADR, $0.5 \mu \mathrm{g} / \mathrm{ml}$ ) for the indicated time periods. Nuclear pro-

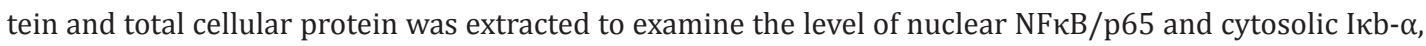
respectively. C - E. Podocytes were pre-treated with the NFKB inhibitor pyrrolidinedithiocarbamate (PDTC; $4 \mu \mathrm{M}$ ) for 30 minutes. ADR (final concentration $0.5 \mu \mathrm{g} / \mathrm{ml}$ ) was then added and incubated for 24 hours in the presence of PDTC. Immunoblot assay was performed to assess the level of nuclear NFKB (C). The abundance of TRPC6 was examined using real time RT-PCR (D) and Western blot assay (E), respectively. Effect of PDTC on cellular apoptosis in wild-type (CTL) and ADR-treated podocyte was assessed using FITC-Annexin V and propidium iodide staining by flow cytometry (F). A - E: three individual experiments were performed, and the representative blots were provided. $\mathrm{F}: \mathrm{n}=3$ independent experiments and triplicates were used for each experiment.

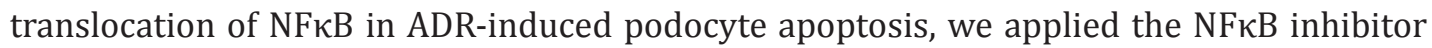
PDTC to ADR-treated podocytes. At 24 hours after ADR treatment, the nuclear NFkB/p65 was significantly inhibited by the application of PDTC (Fig. 3C). We further investigated the effects of NFKB inhibition on TRPC6 expression in ADR-treated podocytes. Real time RTPCR and Western blot revealed that ADR-induced up-regulation of TRPC6 both at mRNA and protein level was dramatically prevented by PDTC (Fig. 3D,E). Of note, the administration of PDTC significantly decreased ( $7.6 \pm 0.81$ vs $12.7 \pm 0.77 ; \mathrm{p}<0.01$ ) cellular apoptosis induced by ADR at 24 hours although the percentage of cellular apoptosis was still higher $(7.6 \pm 0.81$ vs $3.0 \pm 1.0 ; p<0.001$ ) in podocytes treated with ADR and PDTC than control (Fig. 3E). In addition, PDTC showed no significant effect ( $3.2 \pm 0.83$ vs $3.0 \pm 1.0 ; \mathrm{p}>0.05)$ on apoptosis in wild type podocytes (Fig. 3E). These findings imply that NFKB is activated in ADR-treated podocytes, necessary for the up-regulation of TRPC6 expression and induction of cellular apoptosis.

The mTORC2-dependent Akt activation is associated with $N F \kappa B$ activation in ADR-treated podocytes

In prostate cancer cells, Akt-dependent regulation of NFKB is controlled by mTOR [22]. Therefore, we applied the Akt inhibitor triciribine $(20 \mu \mathrm{M})$ to ADR-treated podocytes. Immunoblot assay showed that ADR-induced up-regulation of phospho-Akt ${ }^{\text {Ser473 }}$ was significantly prevented by triciribine, and that the nuclear $\mathrm{NF \kappa B} / \mathrm{p} 65$ level was also decreased in both ADR and triciribine-treated podocytes (Fig. 4A), suggesting that Akt activation may be related to the nuclear translocation of NFKB in ADR-treated podocytes. We also investigated the effects of Akt inhibition on cellular apoptosis induced by ADR in cultured podocytes. The application of TCN alone showed no significant ( $3.4 \pm 0.94$ vs 3.0 $\pm 1.0 ; \mathrm{p}>0.05$ ) effects on apoptosis in wild-type podocytes, while significantly decreased ADR-induced podocyte apoptosis $(9.1 \pm 0.78$ vs $13.9 \pm 0.95$; p < 0.01) (Fig. 4B). Moreover, 
A $\quad C$

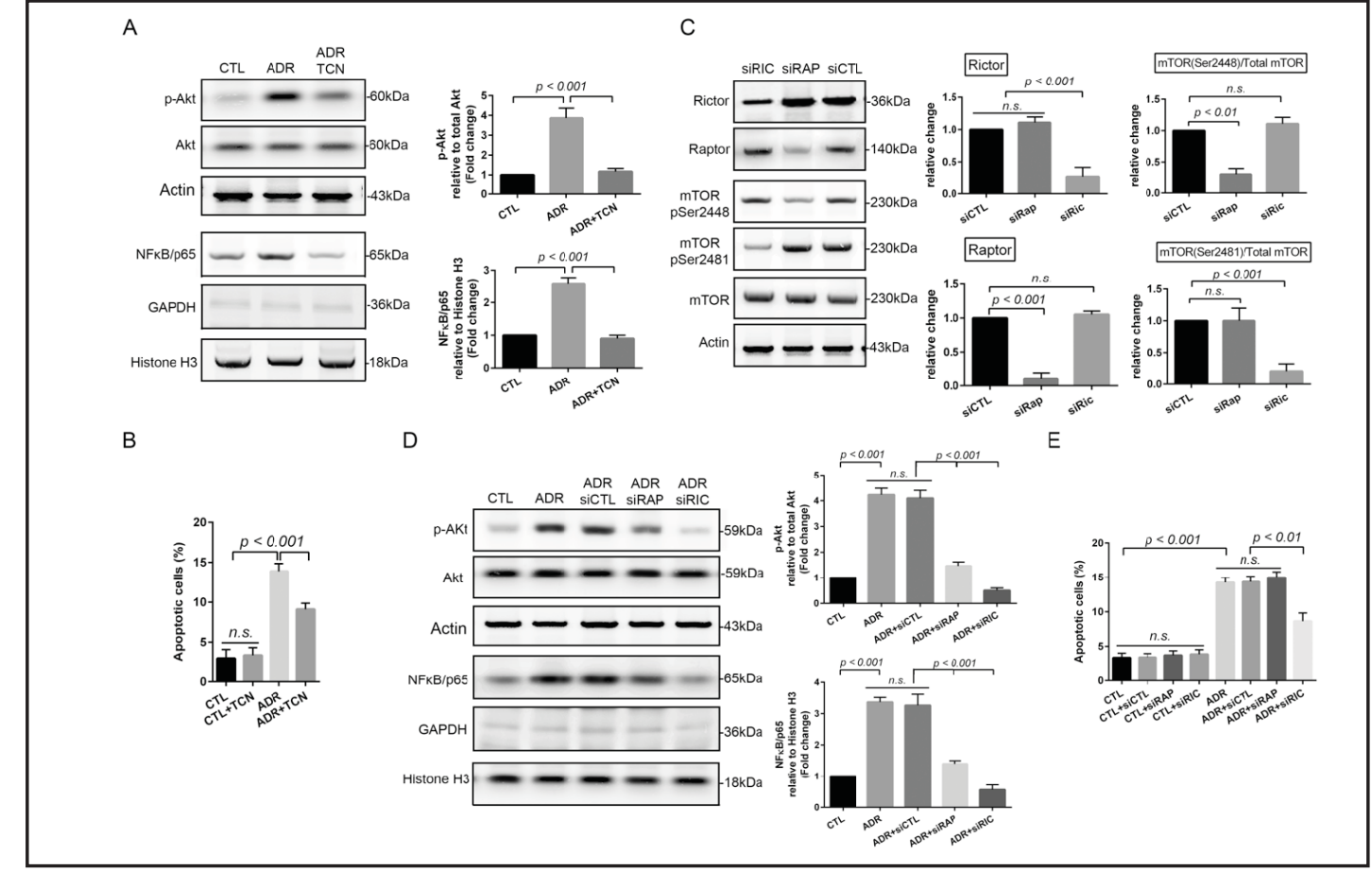

C

Fig. 4. The mTORC2-dependent Akt activation is associated with NFKB translocation in ADR treated podocytes. A, B. Podocytes were pre-treated with the Akt inhibitor triciribine (TCN; $20 \mu \mathrm{M}$ ) for 30 minutes. ADR was then added (final concentration $0.5 \mu \mathrm{g} / \mathrm{ml}$ ) and incubated for 24 hours in the presence of TCN. Immunoblot assay was performed to assess the level of phospho-Akt ${ }^{\text {Ser473 }}$ and nuclear NFkB/p65 (A). Effect of TCN on cellular apoptosis in wild-type (CTL) and ADR-treated podocytes was assessed using FITC-Annexin $\mathrm{V}$ and propidium iodide staining by flow cytometry (B). C - E. To specifically block the mTOR pathway, knockdown of Raptor and Rictor was obtained using siRaptor (siRAP) and siRictor (siRIC), respectively. The control siRNA (siCTL) has no any significant sequence similarity to mouse gene sequences. Expression of mTOR activation was evaluated using anti-phospho-mTOR ${ }^{\text {Ser2448 }}$ and anti-phospho-mTOR ${ }^{\text {Ser2481 }}$ (C). Effect of Raptor and Rictor knockdown on the activation of Akt and nuclear translocation of NFkB was examined using Western blot assay (D). 24 hours after siRNA transfection, cells were treated with Adriamycin (ADR, $0.5 \mu \mathrm{g} / \mathrm{ml}$ ) or cultured media alone (CTL) for another 24 hours (E). A, C, D: three individual experiments were performed, and the representative blots were provided. $\mathrm{B}, \mathrm{E}: \mathrm{n}=3$ independent experiments and triplicates were used for each experiment. n.s.: no significant difference.

we specifically knocked down the expression of the distinct marker of mTOR complexes. Our results revealed that knockdown of Rictor and Raptor specifically down-regulated the level of phospho-mTOR ${ }^{\text {Ser2481 }}$ and phospho-mTOR ${ }^{\text {Ser2448, }}$, respectively (Fig. 4C). Furthermore, effect of Raptor and Rictor knockdown on the activation of Akt and nuclear translocation of NFKB/ p65 was examined in ADR-treated podocytes. Notably, we found that Rictor knockdown, but not Raptor knockdown, significantly prevented the increase of phospho-Akt ${ }^{\mathrm{Ser} 473}$ and nuclear NFKB/p65 induced by ADR treatment (Fig. 4D). Knockdown of Rictor, not Raptor, significantly decreased ( $8.7 \pm 1.2$ vs $14.3 \pm 0.71 ; \mathrm{p}<0.01)$ ADR-induced podocytes apoptosis (Fig. 4E), while Rictor and Raptor knockdown alone showed no significant effect (Rictor: 3.9 \pm 0.65 , Raptor: $3.7 \pm 0.63$ vs control: $3.4 \pm 0.65$; $p>0.05$ ) on cellular apoptosis in wild type podocytes (Fig. 4E).

The increased $\mathrm{Ca}^{2+}$ influx is mediated by TRPC6 in ADR-treated podocytes

Up-regulation of TRPC6 was detected in ADR-induced podocyte apoptosis. TRPC6 is a transient receptor potential ion channel. Activation of TRPC6 can induce the entry of $\mathrm{Ca}^{2+}$ into the cell, while excessive entry of $\mathrm{Ca}^{2+}$ into a cell may induce damage or even cause it to 
Fig. 5. The increased influx of the cytosolic free calcium is mediated by TRPC6 in ADR-treated podocytes. A, B: Knockdown of TRPC6 (TRPC6 KD) was obtained by introducing the siRNA specifically targeting to mouse TRPC6 in wild-type podocytes (WT). C, D: 24 hours after introduction of TRPC6 siRNA, ADR was added (final concentration $0.5 \mu \mathrm{g} / \mathrm{ml}$ ) and incubated for another 24 hours. The control siRNA (CTL KD) has no any significant sequence similarity to mouse gene sequences. The baseline of the intracellular free $\mathrm{Ca}^{2+}$ level was measured with the indicator Fluo-3AM as described in Methods. Activation of TRPC6 was then

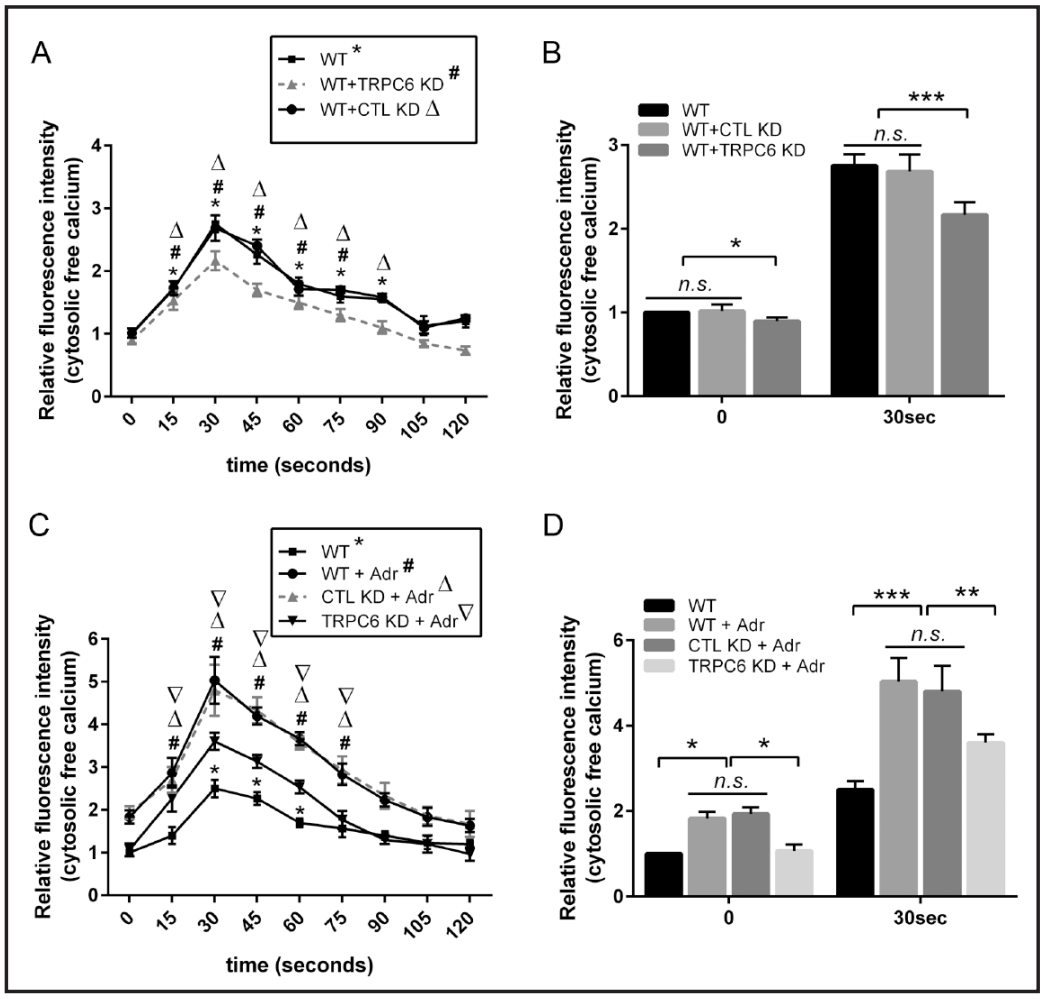
initiated by addition of $10 \mu \mathrm{M}$ of hyperforin, and the intracellular free $\mathrm{Ca}^{2+}$ level was recorded for 2 minutes at a 15 -second interval. A, C: ${ }^{*}, \#, \Delta, \mathbf{\nabla}: \mathrm{p}<0.05$ vs 0 min in the same group. B, D: At 30 seconds following addition of hyperforin, the intracellular free $\mathrm{Ca}^{2+}$ level was compared with 0 min. ${ }^{*}: \mathrm{p}<0.05$; **: $\mathrm{p}<0.01$; $* * *$ : $\mathrm{p}<0.001$. n.s.: no significant difference. $\mathrm{n}=3$ independent experiments, and triplicates were used for each experiment.

undergo apoptosis, or death [6, 8]. Here, we examined the functional alteration of TRPC6 by evaluating the cytosolic $\mathrm{Ca}^{2+}$ level with the indicator Fluo-3AM. Firstly, we investigated the effects of TRPC6 knockdown on the cytosolic $\mathrm{Ca}^{2+}$ level in cultured podocytes. To examine if the $\mathrm{Ca}^{2+}$ influx is TRPC6-dependent, the specific TRPC6 agonist hyperforin was applied [10]. Following addition of hyperforin $(10 \mu \mathrm{M})$, we recorded the dynamic change of cytosolic $\mathrm{Ca}^{2+}$ level for 2 minutes at a 15-second interval. Our data showed that the basal $\mathrm{Ca}^{2+}$ level was significantly $(\mathrm{p}<0.05)$ lower in TRPC6 knockdown cells than that in wild-type and control siRNA-transfected podocytes (Fig. 5A,B). After stimulation with hyperforin, the $\mathrm{Ca}^{2+}$ influx was increased significantly $(\mathrm{p}<0.05)$ in wild-type and control siRNA-transfected podocytes, peaking at 30 seconds and persisting to 90 seconds. Increased $\mathrm{Ca}^{2+}$ influx was detected in TRPC6 knockdown cell whereas the cytosolic $\mathrm{Ca}^{2+}$ level was significantly lower $(\mathrm{p}<0.05)$ in TRPC6 knockdown cell than that in wild-type and control siRNA-transfected podocytes, particularly at 30 seconds and only persisting to 75 seconds (Fig. 5A,B). The change of basal intracellular $\mathrm{Ca}^{2+}$ level was further investigated in ADR-treated podocytes with or without TRPC6 knockdown. The cytosolic $\mathrm{Ca}^{2+}$ level was higher significantly $(\mathrm{p}<0.05)$ in podocytes treated by ADR $(0.5 \mu \mathrm{g} / \mathrm{ml})$ for 24 hours than that in untreated wild-type podocyte. ADRinduced increase of cytosolic $\mathrm{Ca}^{2+}$ level was prevented significantly $(\mathrm{p}<0.05)$ by knockdown of TRPC6, but not by control siRNA (Fig. 5C,D). This result suggests that the functional change of TRPC6 may be involved in ADR-induced podocyte damage. In wild-type podocytes, the $\mathrm{Ca}^{2+}$ influx was increased significantly $(\mathrm{p}<0.05)$ since 30 seconds, and then decreased following stimulation with hyperforin (Fig. 5C). In comparison with un-treated wild-type cells, hyperforin increased the $\mathrm{Ca}^{2+}$ influx significantly $(\mathrm{p}<0.05)$ since 15 seconds with a peak of value at 30 seconds in ADR-treated podocytes. Of note, the increased $\mathrm{Ca}^{2+}$ influx 


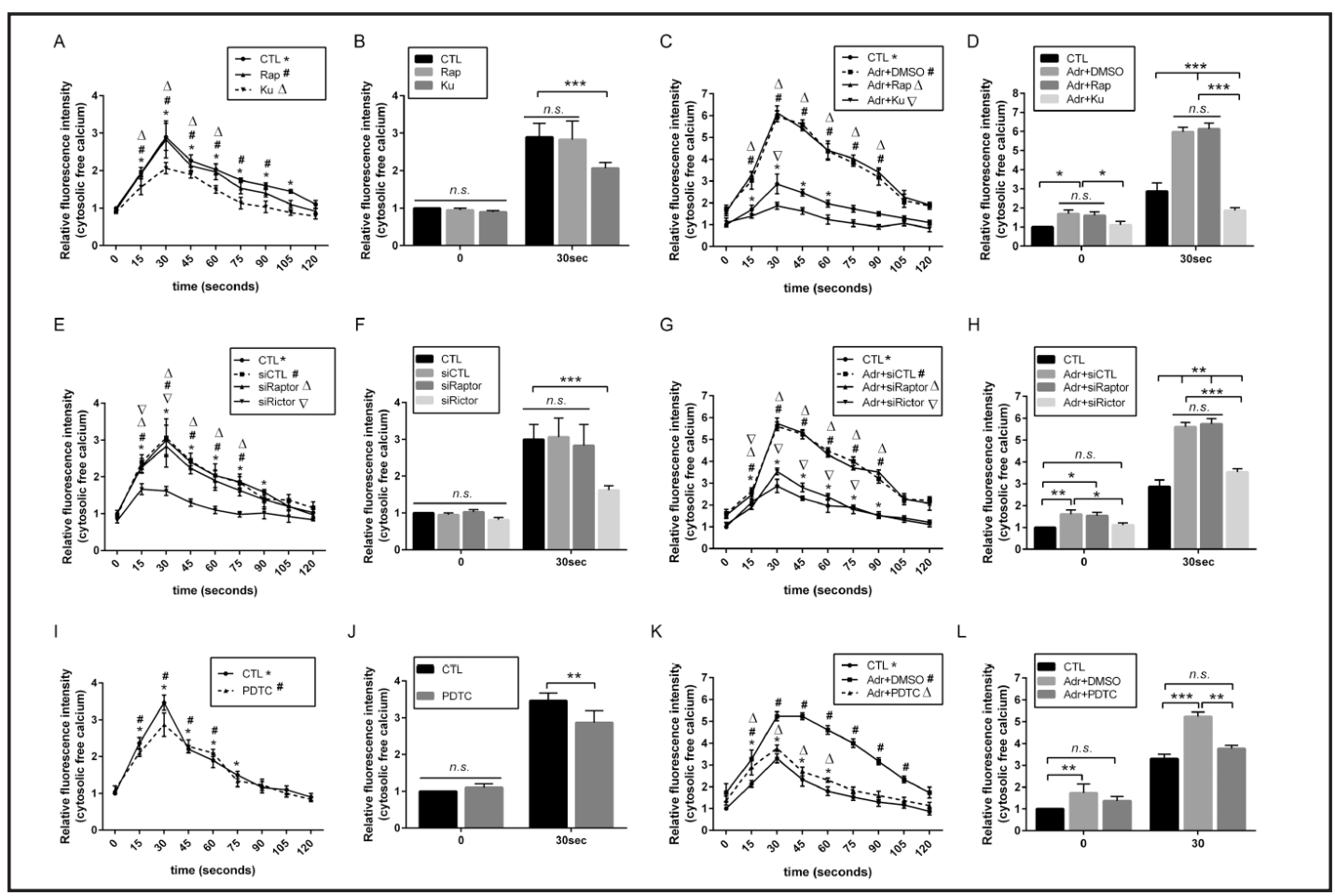

Fig. 6. Inhibition of the mTORC2/Akt/NFkB pathway decreases influx of calcium in ADR-treated podocytes. A - D: Podocytes were pre-treated with rapamycin (Rap; $10 \mathrm{nM}$ ) or ku0063794 (Ku; $1 \mu \mathrm{M})$ for 1 hour. Cells were cultured for 24 hours in the media containing the indicated inhibitors alone (A, B) or both inhibitors and $0.5 \mu \mathrm{g} / \mathrm{ml}$ of ADR (C, D). E - H: Knockdown of Raptor (siRaptor) and Rictor (siRictor) was obtained in podocytes by introducing the validated siRNA specifically targeting to mouse Raptor and Rictor (E, F). 24 hours after introduction of siRNA, ADR was added (final concentration $0.5 \mu \mathrm{g} / \mathrm{ml}$ ) and incubated for another 24 hours (G, H). The control siRNA (CTL KD) has no any significant sequence similarity to mouse gene sequences. I - L: Podocytes were pre-treated with the NFKB inhibitor pyrrolidinedithiocarbamate (PDTC; 4 $\mu \mathrm{M}$ ) for 30 minutes. Cells were cultured for 24 hours in the media containing PDTC alone (I, J) or both PDTC and $0.5 \mu \mathrm{g} / \mathrm{ml}$ of ADR $(\mathrm{K}, \mathrm{L})$. The baseline of the intracellular free $\mathrm{Ca}^{2+}$ level was measured with the indicator Fluo-3AM as described in Methods. Activation of TRPC6 was then initiated by addition of $10 \mu \mathrm{M}$ of hyperforin, and the intracellular free $\mathrm{Ca}^{2+}$ level was recorded for 2 minutes at a 15-second interval. Line graph: *, $\#, \Delta, \boldsymbol{\nabla}: \mathrm{p}<0.05$ vs $0 \mathrm{~min}$ in the same group. Bar graph: At 30 seconds following addition of hyperforin, the intracellular free $\mathrm{Ca}^{2+}$ level was compared with 0 min. ${ }^{*}$ : $\mathrm{p}<0.05$; ${ }^{* *}$ : $\mathrm{p}<0.01{ }^{* * *}$ : $\mathrm{p}<0.001$. n.s.: no significant difference. $\mathrm{n}=3$ independent experiments, and triplicates were used for each experiment.

initiated by hyperforin in ADR-treated podocytes was decreased significantly $(\mathrm{p}<0.05)$ by TRPC6 knockdown at all time points, especially at 30 seconds, while control siRNA showed no obvious effects (Fig. 5C, D). These data imply that induction of TRPC6 may be related to the increase of cytosolic $\mathrm{Ca}^{2+}$ influx in ADR-treated podocytes.

Inhibition of the mTORC2/Akt/NFkB pathway decreases the $\mathrm{Ca}^{2+}$ influx in ADR-treated podocytes

In this study, we also assessed the role of the mTOR/Akt/NFKB pathway on regulation of the $\mathrm{Ca}^{2+}$ influx by using distinguished pathway inhibitors in ADR-treated podocytes. Firstly, we investigated the effects of mTOR inhibition on the cytosolic $\mathrm{Ca}^{2+}$ level in cultured podocytes. Ku0063794 $(1 \mu \mathrm{M})$, the dual inhibitor of both mTORC1 and mTORC2, and rapamycin $(10 \mathrm{nM})$, the specific mTORC1 inhibitor, were used in this study. We found that the basal $\mathrm{Ca}^{2+}$ level showed no significant difference $(\mathrm{p}>0.05)$ between control and ku0063794or rapamycin-treated podocytes (Fig. 6A,B). After stimulation with hyperforin, the $\mathrm{Ca}^{2+}$ influx 


\section{Cellular Physiology Cell Physiol Biochem 2016;40:1079-1093 \begin{tabular}{l|l|l} 
and Biochemistry Published $10.1159 / 000453163$ & $\begin{array}{l}\text { C } 2016 \text { The Author(s). Published by S. Karger AG, Basel } \\
\text { www.karger.com/cpb }\end{array}$
\end{tabular}

Fig. 7. The schematic of the potential signaling pathways related to ADR-induced podocyte damage. Increased TRPC6 expression and channel activity $\left(\mathrm{Ca}^{2+}\right.$ influx) contributes to ADR-induced podocyte apoptosis, which is prevented by the application of ku0063794, triciribine, PDTC, siRictor, and siTRPC6, respectively. Hence, the activation of mTORC2/Akt/ NFkB signaling pathway is associated with TRPC6 induction and apoptosis in ADR-treated podocytes. ADR: Adriamycin; Rap: rapamycin, the mTORC1 inhibitor; Ku: ku0063794, the dual mTORC1 and mTORC2 inhibitor; TCN: triciribine, an Akt inhibitor; PDTC: a NFkB inhibitor.

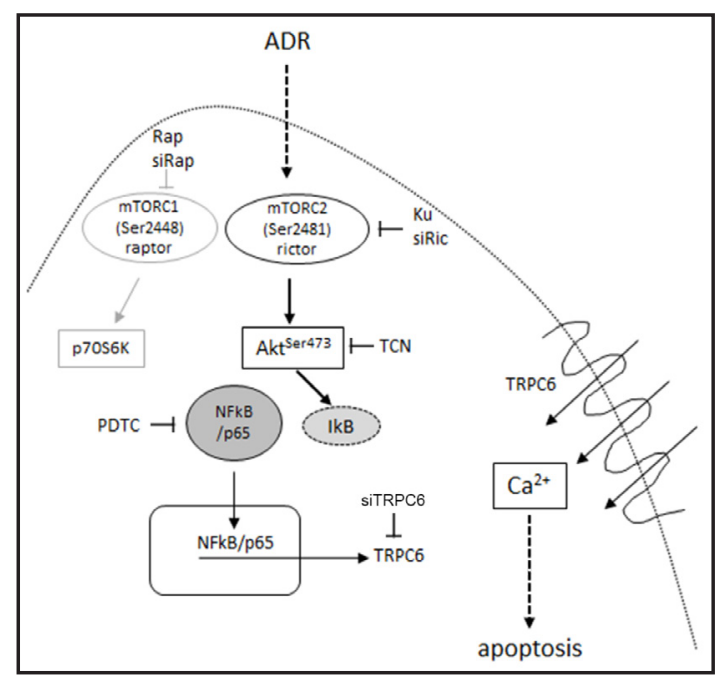

was increased significantly $(\mathrm{p}<0.05)$ in both control and rapamycin or ku0063794-treated podocytes. Nevertheless, the increased $\mathrm{Ca}^{2+}$ influx was significantly lower $(\mathrm{p}<0.05)$ in ku0063794-treated cells than that in control and rapamycin-treated podocytes, particularly at the peaking time 30 seconds (Fig. $6 \mathrm{~A}, \mathrm{~B})$. In podocytes treated with ADR $(0.5 \mu \mathrm{g} / \mathrm{ml})$ for 24 hours, the influx of $\mathrm{Ca}^{2+}$ was increased significantly $(\mathrm{p}<0.05)$ following stimulation with hyperforin $(10 \mu \mathrm{M})$, which was prevented significantly $(\mathrm{p}<0.05)$ by ku0063794 (Fig. 6C,D). However, treatment with rapamycin $(10 \mathrm{nM})$ did not change the hyperforin-induced $\mathrm{Ca}^{2+}$ influx in ADR-treated podocytes (Fig. 6C,D).

Similarly, we found that the basal $\mathrm{Ca}^{2+}$ level showed no significant difference ( $\mathrm{p}>$ 0.05 ) between control and Raptor- or Rictor- knockdown podocytes (Fig. 6E,F). After stimulation with hyperforin, the increased $\mathrm{Ca}^{2+}$ influx was significantly lower $(\mathrm{p}<0.05)$ in Rictor siRNA-transfected cells than that in control and Raptor siRNA-transfected podocytes, particularly at the peaking time 30 seconds (Fig. 6E,F). Moreover, knockdown of Rictor, the marker of mTORC2 complex, inhibited the $\mathrm{Ca}^{2+}$ influx significantly $(\mathrm{p}<0.05)$ in ADR-treated podocytes in comparison with control siRNA, while knockdown of Raptor, the marker of mTORC1 complex, showed no obvious influence on the $\mathrm{Ca}^{2+}$ influx in ADR-treated podocytes (Fig. 6G,H). Therefore, these findings suggest that the mTORC2 complex may be related to the increased influx of $\mathrm{Ca}^{2+}$ in ADR-induced podocyte injury.

Additionally, we examined the effects of the NFKB inhibitor PDTC $(4 \mu \mathrm{M})$ on the $\mathrm{Ca}^{2+}$ influx in ADR-treated podocytes. The basal $\mathrm{Ca}^{2+}$ level showed no significant difference ( $\mathrm{p}$ $>0.05$ ) between control and PDTC-treated podocytes (Fig. 6I,J). After stimulation with hyperforin, the increased $\mathrm{Ca}^{2+}$ influx was slightly but significantly lower $(\mathrm{p}<0.05)$ only at 30 seconds in PDTC-treated cells than that in control podocytes (Fig. 6I,J). Notably, we found that the application of PDTC significantly $(\mathrm{p}<0.05)$ decreased the $\mathrm{Ca}^{2+}$ influx induced by ADR (Fig. 6K,L).

\section{Discussion}

Adriamycin (ADR), an anthracycline antibiotic, is a podocyte toxin used to induce experimental FSGS [23]. Time-dependent increase of podocyte apoptosis was detected in ADR-treated podocytes (Fig. 1A). Notably, ADR treatment significantly enhanced TRPC6 expression (Fig. 1B, C), which was also reported by other groups [19, 24]. Our data from knockdown assay showed that TRPC6 knockdown only marginally reduced ADR-induced podocyte apoptosis (Fig. 1D-F). ADR-induced increase of cleaved caspase3 was also partially inhibited by TRPC6 knockdown (Fig. 1G). This suggests that TRC6 may contribute and there may be other unknown factors that may mediate ADR-induced apoptosis. Therefore, the role 
of TRPC6 in injured podocytes needs be further investigated in other podocyte injury model. TRPC proteins, which belong to the larger TRP superfamily of channels, form $\mathrm{Ca}^{2+}$-permeable channel that are important players in the pathogenesis of renal and cardiovascular diseases [6 - 9]. A gain-of-function mutation in TRPC6 is associated with the onset of hereditary FSGS [6 9]. TRPC6 has been functionally and pharmacologically shown to be involved in $\mathrm{Ca}^{2+}$ influx in podocytes $[25,26]$. In this study, we applied hyperforin and evaluated the functional channel activity of TRPC6 in ADR-treated podocyte with or without TRPC6 knockdown. Hyperforin is a specific agonist of TRPC6, which elevates the intracellular $\mathrm{Ca}^{2+}$ level by activating TRPC6 channels without activating the other isoforms [14]. Firstly, our data showed that hyperforin application significantly resulted in $\mathrm{Ca}^{2+}$ influx in wild-type podocytes, while hyperforininduced $\mathrm{Ca}^{2+}$ influx was decreased significantly in TRPC6 knockdown cells (Fig. 5A, B). This result displayed a baseline TRPC6 activity in podocytes as reported previously $[3,10,11,14]$. We then found that ADR significantly increased both the basal intracellular $\mathrm{Ca}^{2+}$ level and hyperforin-induced $\mathrm{Ca}^{2+}$ influx in podocytes, which was inhibited by TRPC6 knockdown (Fig. 5C, D), suggesting that functional activation of TRPC6 is related to ADR-induced podocyte damage.

We further examined how TRPC6 is induced in ADR-treated podocytes. NFkB, a protein complex that controls transcription of DNA, cytokine production and cell survival, is involved in many biological processes such as inflammation, immunity, differentiation, cell growth, tumorigenesis and apoptosis [20]. NFKB is a homo- or hetero-dimeric complex formed by the Rel-like domain-containing proteins RelA/p65, RelB, NFkB1/p105, NFKB1/ p50, Rel and NFкB2/p52 and the heterodimeric p65-p50 complex [20]. In a conventional

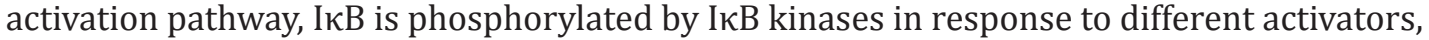
subsequently degraded thus liberating the active NFKB complex which then translocate to the nucleus and bind to target genes [20]. In Ang II-induced podocyte apoptosis, the nuclear translocation of $\mathrm{NF \kappa B} / \mathrm{p} 65$ was detected [10]. Similarly, the nuclear translocation of NFkB was detected in ADR-treated podocytes, which was supported by reduced cytosolic I $\mathrm{B} \alpha$ (Fig. 3A, B). Podocyte-specific NFKB inhibition ameliorates proteinuria in ADR-induced mice nephropathy [27]. Consistently, the application of $\mathrm{NFKB}$ inhibitor PDTC significantly prevented increase of TRPC6 in ADR-treated podocytes (Fig. 3C-E). Our results also show that PDTC decreased TRPC6 channel activity and cellular apoptosis in ADR-treated podocytes (Fig. 6K,L; Fig. 3F). This suggests that ADR-induced upregulation of TRPC6 is NFKB-dependent.

Previous studies suggest that Akt regulates transcriptional activity of NFKB by inducing phosphorylation and subsequent degradation of IKB [28, 29]. In this study, we applied Akt inhibitor triciribine [30], and found that Akt inhibition decreased ADR-induced nuclear translocation of NFKB and cellular apoptosis (Fig. 4A, B). A key effector of Akt-induced signaling is the regulatory protein mTOR. The mTOR downstream from Akt controls NFKB activity in PTEN-inactive prostate cancer cells via interaction with IkB kinase. The mTORC1-associated protein Raptor is required for the ability of Akt to induce NFKB activity [29]. Akt-dependent activation of mTORC1 can affect transcription of p70S6K or 4EBP1. In many cancers, this pathway is overactive, thus reducing apoptosis and allowing proliferation. In addition, the mTORC2 can induce activation of Akt at both Ser473 and Ser450 regulating cellular cytoskeleton and metabolism [29]. In this study, the activation of mTORC2 was identified, showing that phospho-mTOR ${ }^{\text {Ser2481 }}$ and phospho-Akt ${ }^{\text {Ser473 }}$ was significantly increased, while there was no obvious change of phospho-mTOR ${ }^{\text {Ser2448 }}$ and phospho-p70S6K levels in ADRtreated podocytes (Fig. 2A, B). It was reported that mTOR was activated by reduced AMPK phosphorylation in high-glucose-induced podocytes apoptosis [31], while it is unclear which mTOR complex is activated. In addition, the mTORC1 is hyper-activated in podocytes from both diabetic mice and patients with diabetes. Nevertheless, complete deletion of mTORC1 activity in podocyte specific Raptor knockout mice leads to early onset of proteinuria [32, 33]. These findings suggest that although over-activated mTORC1 is causative for podocyte injury, the basal mTORC1 activity is required for maintaining the physiologic functions of podocyte. In normal podocyte, knockdown of mTORC2 complex protein Rictor, not mTORC1

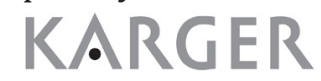




\section{Cellular Physiology Cell Physiol Biochem 2016;40:1079-1093 and BiOChemistry Published online: December 14, $2016 \quad \begin{aligned} & \text { DOI: 10.1159/000453163 } 2016 \text { The Author(s). Published by S. Karger AG, Basel } \\ & \text { www.karger.com/cpb }\end{aligned}$ \\ Zhang et al.: TRPC6 Signaling in ADR-Induced Podocyte Apoptosis}

complex protein Raptor, suppresses TRPC6 expression and $\mathrm{Ca}^{2+}$ influx [14]. Here, we investigated the distinct role of mTOR complex in ADR-treated podocytes. The application of rapamycin, the sensitive inhibitor of mTORC1, did not influence phospho-Akt ${ }^{\mathrm{Ser} 473}$ level (Fig. 2C) and cellular apoptosis following ADR treatment (Fig. 2D), suggesting that Akt activation may be mTORC1-independent. Ku0063794, a dual inhibitor of mTORC1 and mTORC2, was applied in the current study. Our data show that ADR-induced activation of mTORC1 and mTORC2 as well as their downstream effectors including p70S6K and Akt was remarkably inhibited by ku0063794 (Fig. 2C). Furthermore, ADR-induced apoptosis was also decreased by ku0063794 (Fig. 2D), implying that the mTORC2 pathway was involved in ADR-induced podocyte apoptosis.

To further explore the role of mTOR signaling pathways, we specifically knockdowned Raptor and Rictor, respectively. Our data show that only Rictor knockdown prevented ADRinduced activation of Akt and nuclear translocation of NFKB/p65 (Fig. 4C, D). Importantly, we also examined the role of distinguished mTOR complex in regulating TRPC6 channel activity. Rapamycin and Raptor knockdown showed no obvious effects on $\mathrm{Ca}^{2+}$ influx in ADR-treated podocytes, whereas ku0063794 and Rictor knockdown significantly decreased ADR-induced activation of TRPC6 (Fig. 6A-H). In podocyte specific Rictor knockout mice, where mTORC2 activity is abolished in podocytes, did not show any obvious phenotypes [33]. Similarly, we did not observe increase of apoptosis in wild-type podocytes with either TRPC6 knockdown or specific mTOR pathway inhibition (Fig. 1G, Fig. 2D, Fig. 4E). However, upon bovine serum albumin overloading stress, proteinuria level was increased significantly in podocyte Rictor knockout mice compared with control [33], suggesting that the function of mTORC2 in podocytes is required for their adaptation under certain stress conditions.

Taken together, we provide evidence that the mTORC2/Akt/NFkB pathwaymediated activation of TRPC6 may contribute to ADR-induced podocyte apoptosis (Fig. 7). Nevertheless, it has been demonstrated that ADR enhances TRPC6 expression in angiotensin II (AngII)-dependent manner [25]. Furthermore, ADR-induced podocyte damage requires TRPC6-mediated $\mathrm{Ca}^{2+}$ influx and the activation of the $\mathrm{Ca}^{2+}$-dependent protein phosphatase calcineurin and its substrate nuclear factor of activated T cells (NFAT) [25]. In podocytes, it was also reported that insulin increases TRPC6 expression by a calcineurin-dependent pathway [34]. This suggests that a co-activation of both mTORC2/Akt/NFkB and AngII/ calcineurin/NFAT pathways might occur post ADR-induced podocyte injury. On the other hand, activation of Akt leads to inactivation of the effector GSK-3 $\beta$, resulting in degradation of NFAT by the proteasome and subsequent inhibition of cancer cell migration [35]. Therefore, there may be a crosstalk between the two pathways in ADR-induced podocyte injury. The molecular mechanism of ADR-driven TRPC6 expression needs be further investigated to underline the critical role of TRPC6 in the pathogenesis of podocyte injury and proteinuria.

\section{Disclosure Statement}

We declare that we have no conflict of interest.

\section{References}

1 Scott RP, Quaggin SE: Review series: The cell biology of renal filtration. J Cell Biol 2015;209:199-210.

2 Nagata M: Podocyte injury and its consequences. Kidney Int 2016;89:1221-1230.

3 Winn MP, Conlon PJ, Lynn KL, Farrington MK, Creazzo T, Hawkins AF, Daskalakis N, Kwan SY, Ebersviller S, Burchette JL, Pericak-Vance MA, Howell DN, Vance JM, Rosenberg PB: A mutation in the TRPC6 cation channel causes familial focal segmental glomerulosclerosis. Science 2005;308:1801-1804.

4 Walz G: Slit or pore? A mutation of the ion channel TRPC6 causes FSGS. Nephrol Dial Transplant 2005;20:1777-1779. 


\section{Cellular Physiology Cell Physiol Biochem 2016;40:1079-1093 \begin{tabular}{l|l|l} 
and Biochemistry & DOI: 10.1159/000453163 & $\begin{array}{l}\text { C } 2016 \text { The Author(s). Published by S. Karger AG, Basel } \\
\text { www.karger.com/cpb }\end{array}$
\end{tabular} \\ Zhang et al.: TRPC6 Signaling in ADR-Induced Podocyte Apoptosis}

5 Kong F, Ma L, Zou L, Meng K, Ji T, Zhang L, Zhang R, Jiao J: Alpha1 Adrenergic Receptor Activation Stimulates Calcium Entry and Proliferation via TRPC6 Channels in Cultured Human Mesangial Cells. Cell Physiol Biochem 2015;36:1928-1938.

6 Greka A, Mundel P: Balancing calcium signals through TRPC5 and TRPC6 in podocytes. J Am Soc Nephrol 2011;22:1969-1980.

7 Mu YP, Lin DC, Yan FR, Jiao HX, Gui LX, Lin MJ: Alterations in Caveolin-1 Expression and Receptor-Operated Ca2+ Entry in the Aortas of Rats with Pulmonary Hypertension. Cell Physiol Biochem 2016;39:438-452.

8 Ilatovskaya DV, Staruschenko A: TRPC6 channel as an emerging determinant of the podocyte injury susceptibility in kidney diseases. Am J Physiol Renal Physiol 2015;309:F393-F397.

9 Szabó T, Ambrus L, Zákány N, Balla G, Bíró T: Regulation of TRPC6 ion channels in podocytes - Implications for focal segmental glomerulosclerosis and acquired forms of proteinuric diseases. Acta Physiol Hung 2015;102:241-251.

10 Zhang H, Ding J, Fan Q, Liu S: TRPC6 up-regulation in Ang II-induced podocyte apoptosis might result from ERK activation and NF-kappaB translocation. Exp Biol Med (Maywood) 2009;234:1029-1036.

11 Anderson M, Roshanravan H, Khine J, Dryer SE: Angiotensin II activation of TRPC6 channels in rat podocytes requires generation of reactive oxygen species. J Cell Physiol 2014;229:434-442.

12 Wang Z, Wei X, Zhang Y, Ma X, Li B, Zhang S, Du P, Zhang X, Yi F: NADPH oxidase-derived ROS contributes to upregulation of TRPC6 expression in puromycin aminonucleoside-induced podocyte injury. Cell Physiol Biochem 2009;24:619-626.

13 Li Z, Xu J, Xu P, Liu S, Yang Z: Wnt/ $\beta$-catenin signalling pathway mediates high glucose induced cell injury through activation of TRPC6 in podocytes. Cell Prolif 2013;46:76-85.

14 Ding F, Zhang X, Li X, Zhang Y, Li B, Ding J: Mammalian target of rapamycin complex 2 signaling pathway regulates transient receptor potential cation channel 6 in podocytes. PLoS One 2014;9:e112972.

15 Vollenbroker B, George B, Wolfgart M, Saleem MA, Pavenstadt H, Weide T: mTOR regulates expression of slit diaphragm proteins and cytoskeleton structure in podocytes. Am J Physiol Renal Physiol 2009;296:F418-F426.

16 Brosius FC, Coward RJ: Podocytes, signaling pathways, and vascular factors in diabetic kidney disease. Adv Chronic Kidney Dis 2014;21:304-310.

17 Grahammer F, Wanner N, Huber TB: mTOR controls kidney epithelia in health and disease. Nephrol Dial Transplant 2014;29:i9-i18.

18 Yao Y, Inoki K: The role of mechanistic target of rapamycin in maintenance of glomerular epithelial cells. Curr Opin Nephrol Hypertens 2016;25:28-34.

19 Liu Y, Su L, Lin Q, Han Y, You P, Fan Q: Induction of C-Mip by IL-17 Plays an Important Role in AdriamycinInduced Podocyte Damage. Cell Physiol Biochem 2015;36:1274-1290.

20 Rangan G, Wang Y, Harris D: NF-kappaB signalling in chronic kidney disease. Front Biosci (Landmark Ed) 2009;14:3496-3522.

21 Lan HY, Chung AC: TGF- $\beta /$ Smad signaling in kidney disease. Semin Nephrol 2012;32:236-243.

22 Dan HC, Cooper MJ, Cogswell PC, Duncan JA, Ting JP, Baldwin AS: Akt-dependent regulation of NF-\{kappa\}B is controlled by mTOR and Raptor in association with IKK. Genes Dev 2008;22:1490-1500.

23 Fogo AB: Animal models of FSGS: lessons for pathogenesis and treatment. Semin Nephrol 2003;23:161171.

24 Sonneveld R, Ferrè S, Hoenderop JG, Dijkman HB, Berden JH, Bindels RJ, Wetzels JF, van der Vlag J, Nijenhuis T: Vitamin D down-regulates TRPC6 expression in podocyte injury and proteinuric glomerular disease. Am J Pathol 2013;182:1196-1204.

25 Nijenhuis T, Sloan AJ, Hoenderop JG, Flesche J, van Goor H, Kistler AD, Bakker M, Bindels RJ, de Boer RA, Möller CC, Hamming I, Navis G, Wetzels JF, Berden JH, Reiser J, Faul C, van der Vlag J: Angiotensin II contributes to podocyte injury by increasing TRPC6 expression via an NFAT-mediated positive feedback signaling pathway. Am J Pathol 2011;179:1719-1732.

26 Yu L, Lin Q, Liao H, Feng J, Dong X, Ye J: TGF- $\beta 1$ induces podocyte injury through Smad3-ERK-NF- $\kappa B$ pathway and Fyn-dependent TRPC6 phosphorylation. Cell Physiol Biochem 2010;26:869-878.

27 Yamashita M, Yoshida T, Suzuki S, Homma K, Hayashi M: Podocyte-specific NF- $\kappa B$ inhibition ameliorates proteinuria in Adriamycin-induced nephropathy in mice. Clin Exp Nephrol DOI:10.1007/s10157-0161268-6. 


\section{Cellular Physiology Cell Physiol Biochem 2016;40:1079-1093 \begin{tabular}{l|l|l} 
DOI: 10.1159/000453163 & $\begin{array}{l}\text { O 2016 The Author(s). Published by S. Karger AG, Basel } \\
\text { www.karger.com/cph }\end{array}$
\end{tabular} \\ Zhang et al.: TRPC6 Signaling in ADR-Induced Podocyte Apoptosis}

28 Lan A, Du J: Potential role of Akt signaling in chronic kidney disease. Nephrol Dial Transplant 2015;30:385394.

29 Ahmad A, Biersack B, Li Y, Kong D, Bao B, Schobert R, Padhye SB, Sarkar FH: Targeted regulation of PI3K/ Akt/mTOR/NF- $\kappa$ B signaling by indole compounds and their derivatives: mechanistic details and biological implications for cancer therapy. Anticancer Agents Med Chem 2013;13:1002-1013.

30 Tanaka E, Asanuma K, Kim E, Sasaki Y, Oliva Trejo JA, Seki T, Nonaka K, Asao R, Nagai-Hosoe Y, AkibaTakagi M, Hidaka T, Takagi M, Koyanagi A, Mizutani S, Yagita H, Tomino Y: Notch2 activation ameliorates nephrosis. Nat Commun 2014;5:3296.

31 Langer S, Kreutz R, Eisenreich A: Metformin modulates apoptosis and cell signaling of human podocytes under high glucose conditions. J Nephrol 2016;29:765-773.

32 Inoki K, Mori H, Wang J, Suzuki T, Hong S, Yoshida S, Blattner SM, Ikenoue T, Rüegg MA, Hall MN, Kwiatkowski DJ, Rastaldi MP, Huber TB, Kretzler M, Holzman LB, Wiggins RC, Guan KL: mTORC1 activation in podocytes is a critical step in the development of diabetic nephropathy in mice. J Clin Invest 2011;121:2181-2196.

33 Godel M, Hartleben B, Herbach N, Liu S, Zschiedrich S, Lu S, Debreczeni-Mór A, Lindenmeyer MT, Rastaldi MP, Hartleben G, Wiech T, Fornoni A, Nelson RG, Kretzler M, Wanke R, Pavenstädt H, Kerjaschki D, Cohen CD, Hall MN, Rüegg MA, Inoki K, Walz G, Huber TB: Role of mTOR in podocyte function and diabetic nephropathy in humans and mice. J Clin Invest 2011;121:2197-2209.

34 Xia S, Liu Y, Li X, Thilo F, Tepel M: Insulin Increases Expression of TRPC6 Channels in Podocytes by a Calcineurin-Dependent Pathway. Cell Physiol Biochem 2016;38:659-669.

35 Yoeli-Lerner M, Chin YR, Hansen CK, Toker A: Akt/protein kinase b and glycogen synthase kinase-3beta signaling pathway regulates cell migration through the NFAT1 transcription factor. Mol Cancer Res 2009;7:425-432. 